\title{
El sentido de la pregunta por los nombres de Dios en Pseudo Dionisio Areopagita y Clarence Finlayson ${ }^{1}$
}

\author{
Hugo Ochoa \\ INSTITUTO DE FILOSOFÍA \\ PONTIFICIA UNIVERSIDAD CATÓLICA DE VALPARAÍSO \\ hugo.ochoa@pucv.cl
}

Resumen: Pseudo Dionisio Areopagita inicia una larga tradición que interroga por los nombres de Dios, cuestión central para la fe, ya que permite elucidar su contenido y su sentido. No obstante, dar con el verdadero nombre de Dios excede absolutamente las capacidades cognoscitivas del ser humano, de allí que Pseudo Dionisio haya recogido los nombres que Dios recibe, así sea como atributos, de las Sagradas Escrituras. Finlayson se propone un camino completamente distinto, al menos metodológicamente, pues trata de establecer la esencia metafísica de Dios, distinguiéndola de sus atributos, esencia que revelaría los nombres de Dios. Este trabajo pretende mostrar que uno y otro camino se entrecruzan y por ello concuerdan en su propósito: dar un fundamento vital a la fe.

Palabras clave: Pseudo Dionisio, Finlayson, nombres de Dios, fe, teología negativa.

Abstract: Pseudo-Dionisyus the Areopagite is the beginner of a longstanding tradition wherein the question about the names of God rises, which is a core matter of faith, as it permits to elucidate its content and the sense. Nonetheless, to find the real names of God largely exceeds the cognitive abilities of the human being, hence Pseudo-Dionisyus collected the names of God, as attributes in the Scripture. Finlayson, on the other hand, proposes a completely different way, at least from the methodological standpoint, wherein he attempts to establish the metaphysical essence of God, by distinguishing it from its attributes, the essence may reveal the names of God. The purpose of this work is to show that both paths meet and consequently agree in their purpose: lay a vital foundation for the faith.

Key words: Pseudo-Dionisyus, Finlayson, names of God, faith, negative theology.

$\overline{1}$ Este trabajo forma parte del Proyecto FONDECYT 1110432. 


\section{INTRODUCCIÓN}

¿Por qué los nombres de Dios? La fe requiere de contenido y depende de este; una fe no puede estar fundada en la mera afirmación de la existencia, por cuanto la existencia, de suyo, como veremos, no dice relación a contenido alguno ${ }^{2}$. Pero no se trata del contenido doctrinal de la fe, sino de su contenido subjetivo, vale decir, de la presencia misma del objeto de la fe en el creyente. Quién es Dios es, pues, una cuestión central para determinar no solo la índole de la fe, sino que, como principio motivante de la fe misma, también es fundamental para establecer su sentido. Proponerse elucidar los nombres de Dios significa, ciertamente, una desmesura para el entendimiento humano, de allí que lo más pertinente es atenerse a los nombres o atributos que Dios se da en las Sagradas Escrituras, y tal es el camino que emprende Pseudo Dionisio Areopagita.

Por su parte, Tomás de Aquino, aunque refiriéndose permanentemente a las Escrituras, indaga los nombres de Dios a partir de las perfecciones de las creaturas, "según habitud al principio y por vía de eminencia y eliminación" 3 , de modo que la atribución de nombres a Dios se realiza por vía analógica, ya que no es posible nombrar a Dios sino por las creaturas ${ }^{4}$, dado que "en Dios existen las perfecciones de todas las cosas" . Clarence Finlayson intentará alcanzar la esencia divina a partir de los fundamentos de la metafísica de Tomás de Aquino, de quien se declara discípulo fiel. De acuerdo a la metafísica tomista sería necesario establecer una distinción fundamental entre la esencia misma de Dios y sus atributos, los cuales derivan de esta ${ }^{6}$. No obstante lo anterior, no puede tratarse de la esencia divina en sí misma, sino solo quoad nos, de modo que al afirmar una preeminencia, quoad nos, de los que Finlayson designa como constitutivos formales de la divinidad, lo que pretende es retrotraer los atributos a sus principios. Pero, como intentaremos mostrar, estos constitutivos formales de la esencia de Dios, si bien aparen-

2 C. Finlayson, Dios y la Filosofía (Universidad de Antioquia, Medellín 1945) 83.

3 Tomás de Aquino Suma Teológica, q. XIII, a. 1. (Club de Lectores, Buenos Aires 1988).

4 Tomás de Aquino Suma Teológica q. XIII, a. 4.

5 Tomás de Aquino Suma Teológica q. IV, a. 2.

6 C. Finlayson, Dios y la Filosofía, "Estos nombres no constituyen, ni aun quoad nos, atributos sino la propia esencia considerada desde puntos de vista diferentes", $50-51$. 
El sentido de la pregunta por los nombres de Dios en Pseudo Dionisio Areopagita y... 433

temente establecidos en el puro plano metafísico, dan razón de los momentos fundamentales de la fe en una perspectiva vivencial.

\section{Los nombres de Dios en la Revelación según Pseudo Dionisio}

Pseudo Dionisio Areopagita, identificado originalmente con el discípulo que San Pablo habría hecho en Atenas (Hechos 17, 33-34), parece haber sido un monje, probablemente sirio, que vivió entre los años 450 y 520 . Sus escritos tienen una marcada influencia del neoplatonismo, particularmente Plotino y Proclo ${ }^{7}$, y muestra un extraordinario conocimiento de las Sagradas Escrituras. Es autor de Los nombres de Dios, La jerarquía celeste, La jerarquía eclesiástica, La Teología mística y una serie de cartas. El prestigio de estos escritos fue muy grande; la síntesis teológico-mística que allí se encuentra ha sido permanente inspiración para la mística cristiana.

Pseudo Dionisio Areopagita realiza la distinción entre una teología positiva o catafática, y una teología negativa ${ }^{8} \mathrm{o}$ apofática ${ }^{9}$.

"Conviene alabar la negación de modo muy diferente a la afirmación. Afirmar es ir poniendo cosas a partir de principios, bajando por los medios y llegar hasta los últimos extremos. Por la negación, en cambio, es ir quitándolas desde los últimos extremos y subir a los principios. Quitamos todo aquello que impide conocer desnudamente al Incognoscible"10.

Esta concepción de la teología, entendida como camino de vida hacia Dios, tendrá una importancia fundamental, especialmente para la mística. Ambos caminos, el afirmativo y el negarivo, son necesarios para acercarse a Dios, precisamente porque es incognoscible y, no obstante, el ser humano no puede renunciar a su conocimiento. Respecto a su influencia en la mística, Benedicto XVI, refiriéndose a Pseudo Dionisio Areopagita dijo en una audiencia pública: "Hasta esa época para los cris-

7 Y. De Andía, "Neoplatonismo y cristianismo en Pseudo Dionisio Areopagita”, en Anuario Filosófico, 33/2 (2000) 363-394.

8 L. PANIER, "Quelques notes sur la théologie négative -incidences sémiotiques-", en Actes Semiotiques, 115 (2012).

9 Pseudo Dionisio Areopagita, La teología mistica, Obras Completas (BAC, Madrid 2007) 248 ss.

10 Pseudo Dionisio Areopagita, Teología mistica, 248. 
tianos esta palabra [mística] era equivalente a la palabra «sacramental», es decir, lo que pertenece al «mysterion», sacramento. Con él, la palabra «mística» se hace más personal, más íntima: expresa el camino del alma hacia Dios"11.

La realidad de Dios es supra-esencial y secreta, y sobrepasa toda razón, pensamiento y esencia ${ }^{12}$. No obstante, Dios no permanece del todo incomunicable, porque en razón de su propia bondad se manifiesta proporcionalmente a la capacidad de sus creaturas. De allí que en Los nombres divinos Pseudo Dionisio se atenga a los atributos de Dios revelados en la Escritura ${ }^{13}$ como manifestación de sí mismo: Bondad, Belleza, Luz, Vida, Sabiduría, Inteligencia, Unidad, etc. No obstante, el método catafático, ordenado a elucidar lo que cada de uno de estos nombres significa, es necesario completarlo con el apofático, es decir, negar todo límite, toda imperfección que comprenda estos nombres. Dios infinito no se puede reducir a ninguna de esas nominaciones, pues trasciende a todas ellas, por cuanto Dios es propiamente innominable, y así el camino termina, entonces, en una contemplación mística. No obstante, esta está al final del camino, es decir, este debe comenzar necesariamente por la revelación si no quiere caer en un vacío sin sentido, en el que solo se hace presente la soledad humana. "Por eso, de ninguna manera debe uno atreverse a hablar ni a pensar nada sobre la Deidad supraesencial y misteriosa fuera de lo que nos ha sido revelado por las Sagradas Escrituras"14. Con lo cual se establece un límite que es, precisamente, el que de alguna manera Finlayson transgrede.

Toda definición se realiza en virtud de un límite, todo concepto exige un acotamiento, toda aprehensión y sensación solo alcanza lo finito, no obstante, es posible dirigirse a la divinidad por medio de símbolos que, si bien son parciales y no propios para alcanzar las realidades divinas, en la medida que preparan el espíritu y marcan una dirección, son efectivamente un camino. De modo que toda "determinación" de la revelación divina en lo que respecta a sus nombres, debe ser realizada junto con la afirmación de su trascendencia, trascendencia que tiene su fundamento

11 Benedicto XVI, Audiencia general del 14 de mayo de 2008, en https://es.zenit. org/articles/benedicto-xvi-presenta-la-figura-del-pseudo-dionisio-areopagita/

12 Pseudo Dionisio Areopagita, Los nombres de Dios, Obras Completas (BAC, Madrid 2007) 5.

13 Pseudo Dionisio Areopagita, Los nombres de Dios, 17.

14 Pseudo Dionisio Areopagita, Los nombres de Dios, 5. 
El sentido de la pregunta por los nombres de Dios en Pseudo Dionisio Areopagita y... $\mid 435$

en su carácter de causa universal de todo ser y, por lo tanto, tiene que estar más allá de todo ser, porque es "supra-esencial". Pero, al mismo tiempo, esta supra-esencia, como causa de todo ser, es "todo en todos"15, y, por lo tanto, "contiene en sí misma de antemano de manera simple e indefinidamente todos los seres. Por ello es merecidamente celebrada y nombrada por todos los seres" 16 .

La trascendencia, según Psuedo Dionisio, debe ser entendida a partir de una forma de participación ${ }^{17}$, o comunidad entre creador y creatura, ya que Dios es a la vez inmanente y trascendente a su creación, y se trata, pues, de intentar remontarse a su principio. "Participar es recibir algo sin que el que da, pierda algo" ${ }^{18}$, es más, no lo pierde, no solo porque no lo puede perder dada la naturaleza de lo que da, sino sobre todo, dada la "naturaleza" de quien da. Cuando el maestro enseña, es evidente que no pierde nada al participarlo por la naturaleza inmaterial de lo que comunica, pero en el caso de la participación que tienen las creaturas de la divinidad, esta no pierde nada porque en realidad no participa algo sino que se participa a sí mismo, todo es en Él y por Él. Por ello, al comprender la realidad como participación de la supraesencia, Pseudo Dionisio propone recurrir a la analogía ${ }^{19}$ para explicar las verdades divinas a nuestra inteligencia. Participación es el modo como está presente la divinidad en sus creaturas, analogía es el método para intentar comprenderla. La manera de conocerlo es, pues, por desconocimiento ${ }^{20}$.

El recorrido por los nombres divinos debe ser entendido, entonces, como un ejercicio espiritual, una forma de templar las propias fuerzas, porque combate tanto la arrogancia como el exceso de humildad. La lectura de Los nombres divinos debería conducir más allá del entendimiento, más allá de la ciencia, más allá del saber, hacia un estado del espíritu en el que la revelación cobrara vida en la propia vida. De modo que la misma aprehensión del carácter absolutamente trascendente de la divinidad predispondría a quien hace este ejercicio para la trascendencia de sí. Uno de los métodos para esto, tal como Pseudo Dionisio

\footnotetext{
151 Cor. 15, 28.

16 Pseudo Dionisio Areopagita, Los nombres de Dios, 13.

17 Pseudo Dionisio Areopagita, Los nombres de Dios, 21.

18 A., Hubert, "La participación. Meditación desde el aporte de Pseudo Dionisio", en Teología y Vida, 52/1-2, (2011) 258.

19 Pseudo Dionisio Areopagita, Los nombres de Dios 66.

20 Pseudo Dionisio Areopagita, Los nombres de Dios 94.
} 
hace presente la trascendencia, es la contrariedad: Dios es principio y fin, luz y oscuridad, uno y múltiple, grande y pequeño, semejante y desemejante, viejo y niño, está en todo lugar y fuera de todo lugar, etc. La contrariedad pone al entendimiento fuera de sí, ya que la negación no consiste en mostrar que Dios no es esto ni esto otro, sino que es a la vez esto y esto otro. La mera negación de todo arribaría al final a nada; como ninguna de las determinaciones puede agotarlo, la negación de estas no conduce hacia la nada, sino hacia la contraria de cada una de las determinaciones. Dios no solo trasciende toda afirmación, sino que también trasciende toda negación ${ }^{21}$. Así, la teología negativa también opera en virtud de contrarios: Dios no permanece inmóvil ni se mueve, ni es similitud ni disimilitud, ni filiación, ni paternidad, no es nada de aquello que pertenece al no ser, ni nada de lo que pertenece al $\operatorname{ser}^{22}$, porque no es aprehensible por el entendimiento ${ }^{23}$ y este, para entender, o bien afirma, o bien niega: juzga. Su conocimiento se realiza siempre en virtud de un juicio, pero en este caso se trata de lo absolutamente indivisible ${ }^{24}$ y siempre idéntico ${ }^{25}$. Así, pues, se trata de usar precisamente de la separación y unión característica del juicio para poner en evidencia el carácter radicalmente limitado del "es" judicativo propio del lenguaje: "Dios no es según este o aquel modo"26.

Sin embargo, no se trata de renunciar al entendimiento por incapaz de alcanzar el conocimiento de la realidad divina, aunque efectivamente lo trasciende, como tampoco se trata de renunciar a las palabras, que no pueden nombrarlo, ni a la sensibilidad, que no puede percibirlo; si así fuera, la Escritura no tendría sentido. Es necesario entender a la inteligencia como una potencia que intenta rebasarse desde sí misma, a las palabras como símbolos de un misterio, al producto de la sensibilidad como imágenes ${ }^{27}$ que, purificadas, pueden hacer presente lo inefable. La encarnación, el hecho que Dios se haya hecho hombre, revela que el

21 Pseudo Dionisio Areopagita, La teología mistica 250.

22 Pseudo Dionisio Areopagita, La teología mistica 251-252.

23 Pseudo Dionisio Areopagita, Cartas varias, Obras Completas (BAC, Madrid 2007) 255.

24 Pseudo Dionisio Areopagita, Los nombres de Dios 15-16.

25 Pseudo Dionisio Areopagita, Los nombres de Dios 82.

26 Pseudo-Dionisio o Areopagita, Os nomes Divinos, en Obra Completa (Paulus, São Paulo 2004) 79.

27 Pseudo Dionisio Areopagita, Los nombres de Dios 83. 
El sentido de la pregunta por los nombres de Dios en Pseudo Dionisio Areopagita y... 437

camino, si bien trasciende absolutamente lo humano, pasa también por lo humano, la negación de sí mismo es, para el hombre, afirmación de sí. No es posible celebrar a Dios sino a la manera de lo humano ${ }^{28}$. Los conocimientos divinos no iluminan el alma por medio de una intuición intelectual, de la que no somos capaces, sino en virtud de razones discursivas e inductivas ${ }^{29}$. Citando a su maestro Hieroteo dice refiriéndose a Jesús: "estaba en nuestra naturaleza física de forma sobrenatural, y teniendo de forma eminente todas las cosas nuestras de manera superior a nosotros y siendo por nosotros supraesencia de nuestra esencia" 30 , y, de este modo la afirmación de lo humano cobra también el sentido de un llamado hacia sí mismo.

Para Pseudo Dionisio el primero de los nombres de Dios es Bondad o Bien, ya que todo nombre que se le pueda dar corresponde a un don de la bondad divina ${ }^{31}$. Todos los seres deben su ser, su permanencia, su estabilidad, su conservación ${ }^{32}$, el orden que guardan entre ellos, al divino Bien. Esta concepción, de indudable raíz platónica, está claramente matizado por el contenido de las Escrituras. Dios es creador, y este acto significa en realidad que, de una manera a la vez trascendente e inmanente, acoge a la creación en sí mismo, y esto es un acto de suprema bondad; no hay ser fuera del Ser. Por ello la creación es antes que nada un acto de bondad, de acogimiento, contiene en sí toda pluralidad y toda unidad. La bondad trascendente penetra todo ser y es medida de todo $\operatorname{ser}^{33}$. Su obra no es meramente creadora, es deificadora ${ }^{34}$, porque todo lo que es, para ser, debe comunicar en algún sentido con la supraesencial divinidad $^{35}$. Es Bien, además, porque todas las cosas tienden hacia Él, en virtud de la inteligencia las inteligentes, en virtud de la sensibilidad las sensibles, para las que carecen de sensibilidad en virtud de su impulso vital; convierte hacia sí todo lo que ve, todo lo que acaece, todo lo que existe. La conversión a Dios no es meramente intelectual, ni siquiera sensitiva, es existencial, por cuanto la misma existencia es vocación de

\footnotetext{
Pseudo Dionisio Areopagita, Los nombres de Dios 60.

Pseudo Dionisio Areopagita, Los nombres de Dios 38.

Pseudo Dionisio Areopagita, Los nombres de Dios 24.

31 Pseudo Dionisio Areopagita, Los nombres de Dios 26.

32 Pseudo Dionisio Areopagita, Los nombres de Dios 31.

33 Pseudo Dionisio Areopagita, Los nombres de Dios 34.

34 Pseudo Dionisio Areopagita, Los nombres de Dios 25.

35 Pseudo Dionisio Areopagita, Los nombres de Dios 32.
} 
absoluto, de allí que todo lo que es tiende naturalmente a perseverar en el ser. Y este intento se realiza en vistas al fin, que no solo es el pleno y absoluto bien, sino que es la plena y absoluta belleza ${ }^{36}$, porque "produce y mueve a todos los seres y los conserva, dándoles el amoroso deseo de su propia belleza.

Dios es lo bello-bueno, es la fuente de toda armonía, tanto la interna a cada ser como la que relaciona a todos los seres unos con otros. La belleza es claridad, luz, la armonía es orden, unidad, de modo que no solo se trata de una forma de presencia estética, sino que propiamente inteligible, en la medida que claridad y orden son condición necesaria para que el entendimiento alcance su objeto. Pero tampoco se trata solo de la inteligencia o, en general, de las facultades cognoscitivas, la belleza es paz, es acogimiento, es el "y vio Dios que era bueno" del Génesis. De allí que Pseudo Dionisio reúna, ciertamente siguiendo una tradición platónica, a bien y belleza en una unidad como primera expresión de la divinidad. Tanto bien como belleza son lo primero porque ambos convocan, llaman, atraen, y lo hacen "sin salir de si", su sola realidad constituye una forma de presencia que impone y se impone, sin esfuerzo, casi diría con 'dulzura', mueven conmoviendo. Esta fuerza centrípeta, característica del bien y de la belleza, pone en evidencia que ambas son fin y principio ordenador de la totalidad y, de esta manera, se revela el carácter a la vez trascendente e inmanente del fundamento de todo lo que es; "la Hermosura es el origen de todo, como causa eficiente y motora del universo, [...] es el fin de todo y se la ama como causa final, ya que todo debe su origen a la Hermosura, y es modelo porque todo se hace conforme a Ella" ${ }^{37}$. El poder atractivo de lo Bello-Bueno significa, pues, orden, jerarquía, unidad; la mera existencia no revela desde sí al Dios infinito; es la magnificencia, la belleza, el orden admirable de todo en todo, donde se puede encontrar rasgos de lo que trasciende, también, a toda existencia. Ser creatura no es meramente tener una existencia donada, serlo es antes que nada ser figura de la divinidad. Toda creatura es de algún modo símbolo de Dios, en cada una está de manera misteriosa presente la trascendencia o, usando un término kantiano, lo sublime de Dios. "Resumiendo, todo ser existe por el Bien-Hermosura, está en Él

36 L. Clavell, "La belleza en el comentario tomista al De Divinis Nominibus", en Anuario Filosófico 17/2 (1984) 93-99.

37 Pseudo Dionisio Areopagita, Los nombres de Dios 37. 
El sentido de la pregunta por los nombres de Dios en Pseudo Dionisio Areopagita y... 439

y a Él tiende. Gracias al Bien-Hermosura existe y surge todo. Por gracia de Él y en Él existe todo principio ejemplar, final, eficiente, formal, elemental y, en una palabra, todo principio, toda conservación, todo fin" ${ }^{38}$.

La creación surge del "deseo bienhechor", es obra del Bien y, como tal, fruto de la sobreabundancia del Bien, todo lo que hace Este está bien y no puede ser sino bueno. Respecto del mal, Pseudo Dionisio sostiene que aquel no es ser ni está en los seres, el mal es fruto de la impotencia y de la debilidad ${ }^{39}$, y ocurre por abandono o negligencia de la creatura. Se trata, pues, de una carencia, pero la Providencia cuida de cada ser e, incluso, el mal está de algún modo al servicio del bien. En resumen, las limitaciones de las creaturas no suponen un mal, la finitud misma es un bien, aunque limitado, precisamente porque es el modo como las creaturas participan del bien ${ }^{40}$.

"El nombre divino 'Bien', en efecto revela las procesiones todas de la Causa Universal que se extiende hasta el ser y el no-ser y trasciende al ser y al no-ser. En nombre 'ser' se extiende a todos los seres y trasciende a todos" 31 .

De modo que el nombre 'Bien' es más amplio o, mejor dicho, revela con más precisión, al mismo tiempo que con más amplitud, la identidad divina que el nombre 'Ser', en la medida que da cuenta de la Providencia divina. Ciertamente todo lo que es, es por participación del Ser en sí, por ello para Pseudo Dionisio el Ser es la primera manifestación de la bondad divina ${ }^{42}$, todas las demás participaciones, vida, unidad, sabiduría, etc., se asientan en esta primera participación. No obstante, es el Bien el autor de todo lo que es ${ }^{43}$, porque la existencia de lo creado es de suyo una existencia providencial; no solo todo tiene su origen en el Ser en sí, sino que tiene también en Él su destino, de modo que la existencia consiste en el curso entre estos dos extremos. Por ello, como Dios es comprendido necesariamente a partir de su obra y de su revelación, se manifiesta primera y eminentemente como Bien en sí. Así, pues, "todo

38 Pseudo Dionisio Areopagita, Los nombres de Dios 39.

39 Pseudo Dionisio Areopagita, Los nombres de Dios 57.

40 El tratamiento del mal requeriría una explicación mayor, lo cual exigiría un estudio independiente que excede los límites del presente trabajo.

41 Pseudo Dionisio Areopagita, Los nombres de Dios 59.

42 Pseudo Dionisio Areopagita, Los nombres de Dios 62.

43 Pseudo Dionisio Areopagita, Los nombres de Dios 92. 
ha sido creado y existe por el Bien” ${ }^{44}$, todo tiende hacia Él, movido por un deseo amoroso ${ }^{45}$, o eros, término que Pseudo Dionisio prefiere en vez de amor caritativo, o ágape, por cuanto el amor divino constituye una fuerza que atrae y corresponde efectivamente a ese amor ${ }^{46}$. Si bien Dios es creador por causa de la sobreabundancia de su bondad, esta sobreabundancia es el amor mismo, como deseo bienhechor, y por eso Dios no había de quedarse encerrado en sí mismo, estéril, sino que la creación es obra del amor de Dios, tanto como también lo es la misma Providencia divina ${ }^{47}$. De allí también que Dios sea celoso, y despierte en sus obras también un celo que las hace buscarlo y ordenarse entre sí, según jerarquías $^{48}$ que colaboran entre sí para orientarse al fin último.

Todo tiende al Bien y desea el Bien-Hermosura, pero se trata de un deseo que es abandono de sí, no anhelo de posesión. La luz divina rompe la ignorancia, y hace partícipes a las inteligencias, en primer lugar, del resplandor que les es conveniente ${ }^{49}$, y cuando apetecen más, se les da abundantemente "porque amaron mucho". El amor, incluso, conduce incluso al éxtasis, "al no dejar a los que aman ser de sí mismos, sino del amado" ${ }^{50}$. De este modo se puede decir que el punto de encuentro entre Dios y el ser humano es el amor. Por una parte, la creación es obra del Bien, pero en su dimensión amorosa, por esta misma ordena providencialmente su obra, en tanto fin último de todo ser y, además, ilumina en virtud del amor a la inteligencia para que se dirija hacia su propio bien que es Él mismo. Por otra parte, en razón del mismo carácter de creatura, los seres humanos, aunque no solo ellos, sienten un deseo amoroso que los mueve desde sí mismos hacia el Bien, es este deseo amoroso el verdadero punto de partida de la pregunta que interroga por la divinidad. Por eso para Pseudo Dionisio Dios es antes que nada Bien y Hermosura, porque desde la perspectiva de la creatura el fin se da positivamente, como algo a lo que se tiende, como deseo amoroso; la luz del entendimiento, en cambio, alcanza solo a entrever por vía negativa la

44 Pseudo Dionisio Areopagita, Los nombres de Dios 34.

45 Becerra, Cícero Cunha, Dionísio Pseudo-Areopagita. Mistica e Neoplatonismo (Paulus, São Paulo 2009) 65 ss.

46 Pseudo Dionisio Areopagita, Los nombres de Dios 41.

47 Pseudo Dionisio Areopagita, Los nombres de Dios 42.

48 Pseudo Dionisio Areopagita, Los nombres de Dios 37.

49 Pseudo Dionisio Areopagita, Los nombres de Dios 35.

50 Pseudo Dionisio Areopagita, Los nombres de Dios 42. 
El sentido de la pregunta por los nombres de Dios en Pseudo Dionisio Areopagita y... $\mid 441$

identidad de Dios. La fe tiene lugar, pues, en el encuentro entre el amor divino y el amor humano, creador y creatura, providencia y sentido. De este modo el mismo contenido subjetivo de la fe es aquello por lo que se tiene fe, es el mismo Dios bueno el que por su bondad amorosa se muestra en la fe al creyente.

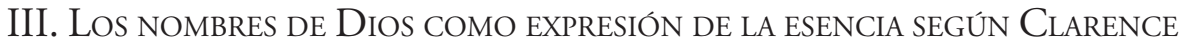 FINLAYSON}

Clarence Finlayson nace en 1913 en Valparaíso, Chile, y muere trágicamente en Santiago en 1954. Licenciado en Filosofía por la Universidad Católica de Chile, obtiene su doctorado en la Universidad de Notre Dame, con una tesis dirigida por Yves Simon y publicada posteriormente por la Universidad de Antioquia con el título Dios y la Filosofía. Finlayson se declara fiel discípulo de Tomás de Aquino, no obstante que en esta obra da un paso que, sin duda, va más allá de los límites fijados por el santo. En su época alcanzó gran reconocimiento, desempeñándose entre los años 1939 y 1942 como profesor en la Universidad de Notre Dame (Indiana) y en 1943 en la Universidad Nacional de México. Entre los años 1943 y 1947 se desempeña como profesor en las universidades Católica Bolivariana y Antioquia de Medellín, donde publica sus obras más importantes. En años siguientes será profesor de la Universidad de Panamá, Visiting Lecturer en la Universidad de Harvard y en la Universidad de North Carolina, profesor en la Universidad Central de Venezuela y luego en la Universidad de Zulia, Maracaibo, para retornar a Chile en 1954, donde se incorpora como profesor en la Universidad Católica de Chile, pero muere ese mismo año.

Si Pseudo Dionisio aborda el problema de Dios desde una perspectiva, se podría llamar, hermenéutica a partir de la Sagradas Escrituras, Clarence Finlayson lo hace desde una perspectiva estrictamente metafísica y, aunque se apoye también en las Sagradas Escrituras, esto lo hace como corroboración de las tesis metafísicas que propone. La razón humana no solo puede, sino que debe abordar el problema de los nombres de Dios, si bien su solución quedará siempre dentro de los límites de lo humano, lo cual significa que solo logrará entrever el misterio. Y debe abordarlo porque, según Finlayson, es la clave central de toda metafísica, se afirme o se niegue la existencia de Dios, ya que en el modo de abordar 
este problema y en su respuesta, entra en juego no solo qué sea ser, sino también lo que signifique bien, deber, libertad, amor, sociedad, cultura, etc., ya que esta pregunta interroga por el límite de lo humano, y así, en definitiva, lo define.

"El problema de la esencia metafísica de Dios es la más alta y misteriosa pregunta que puede hacerse la inteligencia sobre Dios, y en el fondo sobre la realidad en general. En ella están comprendidos todos los problemas constitucionales de la Metafísica. A ella concurren en su solución y en la afirmación misma de la investigación todas las grandes tesis de la ontología" 51 .

Si bien el problema queda teóricamente planteado en términos de qué sea "la esencia metafísica" de Dios, de partida, este planteamiento sufre un giro fundamental: se trata, una vez más, de los nombres de Dios. De modo que hay una identidad no tematizada por Finlayson, entre el nombre y la esencia; sin embargo, la tesis implícita en esta identificación es que la inteligencia humana, y de eso se trata, se apropia intelectualmente de la realidad nombrándola. La misma revelación de Dios en la Escritura se realiza por la intermediación de la palabra, de modo que no puede constituir un sinsentido intentar dar con el nombre divino. No obstante, Finlayson pretende alcanzar nombres que den con la identidad divina misma, es decir, nombres que revelen a Dios en su divinidad, aunque esto solo se pueda alcanzar analógicamente.

Finlayson sostiene que se puede conocer lo que es absolutamente esencial a Dios, en cuanto es la primera causa de todas las cosas y que excede todos sus efectos, por cuanto las ultrasupera ${ }^{52}$. Para ello es necesario que el nombre cumpla copulativamente con tres requisitos: que sea propio y exclusivo de Dios, que sea aquello que lo distingue radical y esencialmente de las creaturas, y que a partir de él se puedan deducir todos los atributos de $\operatorname{Dios}^{53}$. Es decir, la intención de Finlayson no es establecer 'atributos ${ }^{54}$, sino de dar con la fuente de estos, y tal es, escolásticamente hablando, la esencia que, en este caso, no puede darse en una definición, de allí que se busque su nombre. No obstante, como

\footnotetext{
51 C. Finlayson, Dios y la Filosofía 11.

52 Tomás de Aquino Suma Teológica, q. XII, a. 12.

53 C. Finlayson, Dios y la Filosofía 38-39.

54 "Estos nombres no constituyen, ni aun quoad nos atributos, sino la propia esencia considerada desde puntos de vista distintos." C. Finlayson, Dios y la Filosofía, 51.
} 
El sentido de la pregunta por los nombres de Dios en Pseudo Dionisio Areopagita y... 443

dice Tomás de Aquino, "los nombres que le damos [a Dios] no pueden expresar su esencia, tal como es en sî" ${ }^{\prime 5}$. No se trata, pues, de dar con el nombre de Dios en sí mismo, sino tal como puede mostrarse a los seres humanos: "el nombre de Dios en El Mismo es incomunicable e incomprensible, es su absoluto e infinito secreto" 56 . Pero ahora, a diferencia de Pseudo Dionisio, se trata de una exploración cuyo punto de partida es el mismo ser humano, su inteligencia, su vida, su ser, y no la palabra de Dios, aunque todo lo que se diga deba estar refrendada por ella.

Finlayson comienza por establecer las diferentes perspectivas desde las que se puede abordar cuál sea la esencia de la divinidad.

1. Desde el punto de vista de la línea entitativa como tal, de la 'esencia' considerada en sí misma en cuanto 'especificidad'.

2. Desde el punto de vista de la 'naturaleza', es decir de la esencia considerada formalmente como principio de operaciones.

3. Desde el punto de vista de la superabundancia de ser, de la 'gloria'. Quoad nos, este punto de vista expresa la actividad del ser en cuanto terminativo o terminal, en su superabundancia última y por excelencia ${ }^{57}$.

Finlayson considera que esta distinción es fundamental, tanto así que no hacerla induce a confusiones y errores teológicos y metafísicos en los que se ha visto envuelta la escolástica. Estas tres perspectivas darían cuenta, cada una, de una identidad esencial de la Divinidad, los atributos que dimanen de estos nombres esenciales, si bien no difieren ni pueden diferir de la identidad misma de Dios, se fundan o se explican a partir de lo determinado por estas perspectivas. Asimismo, es necesario fijar la formalidad desde la que se plantea el problema, por cuanto el entendimiento humano, desde sí mismo, no puede sino aprehender una 'especificidad', es decir, alcanza su objeto formalmente. Las dos primeras perspectivas interrogan por la esencia y por la naturaleza que, clásicamente constituyen de hecho una identidad en los objetos naturales. Pero la tercera perspectiva se separa, parece, de un análisis puramente metafísico y, por ello, pone en evidencia el límite de la pretensión de alcanzar el nombre de Dios con una perspectiva de esta naturaleza. Por ello, si

55 Tomás de Aquino Suma Teológica, q. XIII. a. 1.

56 C. Finlayson, Dios y la Filosofía 86.

57 C. Finlayson, Dios y la Filosofía 12. 
nuestro conocimiento es, como señala también Finlayson, "por vía de negación y de analogía, de atribución y de trascendencia" ${ }^{58}$, entonces es necesario dar cuenta también, de alguna manera, del fundamento de esa trascendencia; tal sería la gloria, entendida como el 'desborde' que Dios hace de sí mismo. Como trataremos de mostrar, es en esta tercera perspectiva donde las otras dos se encuentran. Cabe recordar que Tomás de Aquino insiste en que para alcanzar un conocimiento de la esencia de Dios, se requiere del auxilio de su propia luz ${ }^{59}$.

Según Finlayson, desde el primer punto de vista, Dios se muestra como ipsum esse subsistens. En esto sigue una larga tradición que arranca del Éxodo, y que, como muestra Finlayson, ha sido recogida por toda la tradición escolástica. Pero, para comprender qué significa esta afirmación, es necesario establecer qué es ser y qué es esencia. Respecto de lo segundo, citando a De ente et essentia, sostiene que "esencia es aquello en lo cual y por lo cual el ente tiene ser" ${ }^{\prime \prime}$, y citando al Padre del Prado esencia es "aquello cuyo acto es ser" ${ }^{61}$. Es decir, la esencia específica al ente a ser 'esto', de allí que sea el término propio del entendimiento, de modo que existe universalmente en el entendimiento y singularmente en la realidad. La esencia, en su consideración puramente intelectiva, dado su carácter universal, aparece como independiente de su existir. No obstante, la plenitud de la esencia es el existir, de modo que la esencia tiene esta doble referencia, por un lado es orden a la existencia y, por otro, al entendimiento, pero en su dimensión puramente inteligible no está sujeta a las contingencias de lo que acaece. La relación de la esencia con la existencia, en las creaturas, no obstante, no es nunca necesaria, ontológicamente siempre está abierta la posibilidad de dejar de ser.

Si bien solo el contenido esencial es directamente inteligible, la existencia se comporta respecto del entendimiento como cierta especie o quididad $^{62}$, pero, porque pone a la esencia fuera del puro orden de la inteligibilidad, contiene cierto aspecto no inteligible. De modo que en todo caso el acceso a lo inteligible del ser se realiza en virtud de la esencia; y su acto -la existencia- que, en el caso de las creaturas, no es acto

\footnotetext{
58 C. Finlayson, Dios y la Filosofía 39.

59 Tomás de Aquino Suma Teológica, q. XII, a. 2.

60 C. Finlayson, Dios y la Filosofía 14.

61 C. Finlayson, Dios y la Filosofía 15.

62 C. Finlayson, Dios y la Filosofía 17.
} 
El sentido de la pregunta por los nombres de Dios en Pseudo Dionisio Areopagita y... $\mid 445$

del acto mismo, sino de la esencia, si bien actualiza un contenido, no es un contenido: ser es acto. Citando De Potentia (q. 7, a. 2, ad 9) "ser es la actualidad de todo acto, y perfección de toda perfección, y no es nada formal"63. Contra Molina, Finlayson afirma que el existir no es un accidente predicamental, como si fuera una forma peculiar de ser de la esencia, por cuanto el existir se distingue realmente de la esencia, es decir, a parte rei ${ }^{64}$. El existir, como acto perfeccionador de la esencia, no puede surgir de esta última, sino que ha de advenirle extrínsecamente, comprendiéndola en su totalidad y en un acto único. Todo lo anterior sigue rigurosamente el pensamiento tomista, pero, a partir de ello mismo, no parece posible alcanzar el propósito enunciado: establecer el nombre de Dios que dé cuenta cabal de su esencia. Efectivamente, si la esencia limita al ser, si el entendimiento solo puede conocer en virtud de una abstracción, si la esencia está siempre abierta a la posibilidad de no ser, si la existencia carece de contenido, etc., entonces la conclusión no podría ser sino que la esencia de Dios y, por lo tanto, su nombre son incognoscibles. Aunque se diga que se trata de la esencia o del nombre quoad nos, aun así, el propósito excede los límites del entendimiento humano, y este tendría que negarse a sí mismo para ponerse en disposición de vislumbrar la esencia de Dios, porque no se trata meramente de dar cuenta de algunos atributos de Dios, sino de su esencia.

En este punto, como lo señala Atria ${ }^{65}$, Finlayson se aparta de la filosofía tomista. Efectivamente, para Tomás de Aquino los nombres que se le atribuyen a Dios son impropios, en la medida que son realizados por vía de eminencia y eliminación y por ello no pueden expresar su esencia $^{66}$, incluso "el que es" es solo el nombre más propio ${ }^{67}$. Atria sostiene que en este punto Finlayson recurre a Escoto, ya que para este el constitutivo formal de la divinidad es la infinitud ${ }^{68}$, y para traer algo a la existencia desde una infinidad de posibles se requiere un ser infinito y una voluntad infinita, y para conocerlos, una inteligencia infinita. A lo cual se agrega la concepción unívoca del ser que tiene Escoto, por

\footnotetext{
63 C. Finlayson, Dios y la Filosofía 23.

64 C. Finlayson, Dios y la Filosofía 25.

65 M. Atria, "Clarence Finlayson y los nombres de Dios", en Academia 13-14, (1986) 132.

66 Tomás de Aquino Suma Teológica, q. XIII, a. 1.

67 Tomás de Aquino Suma Teológica, q. XIII, a. 11.

68 C. Finlayson, Dios y la Filosofía, 56.
} 
cuanto, salvada la distancia infinita que media entre el ser creador y el ser creatura, los conceptos abstraídos de las creaturas pueden aplicarse propiamente a Dios. No obstante, nos parece que la argumentación de Escoto se sostiene en la univocidad, tanto del ser como del conocimiento de Dios, y para Finlayson el ser es análogo y también lo es, por lo tanto, el conocimiento que el ser humano puede tener de Dios. De modo que, si bien en este punto se aparta de Tomás de Aquino, no lo hace en una deriva escotista, sino que intenta conducir el pensamiento de Tomás de Aquino más allá de sí mismo, aunque fundado en él. Por otra parte, Tomás de Aquino sostiene que el carácter infinito de la divinidad es consecuencia de que Dios es el ser subsistente ${ }^{69}$. Ahora bien, el fundamento de la finitud de los seres, según el aquinate radica en la distinción real entre esencia y existencia característica esta de toda creatura, y como en Dios no se da esta distinción, ya que Él es su misma existencia, carece de límites, es infinito.

Los principios de los que parte Finlayson son estrictamente tomistas. Siendo el ser acto de la esencia, esta lo limita y, si bien no tiene sentido preguntar por qué los entes son limitados, sí lo tiene preguntar por qué el ser se limita en los entes, ya que esto es lo que significa la distinción real de ser y esencia; el ser es limitado por una realidad distinta de su actualidad. El existir en sí mismo carece de todo principio de limitación y, por ello, la existencia es más perfecta que la esencia, porque la existencia se compara a la esencia como el acto segundo al acto primero, es decir, es el acto que completa y plenifica a la esencia. Ahora bien, el acto de existir no puede provenir de la esencia, sino que tiene que "provenir solo y necesariamente de un acto, de una actualidad especial y eficasísima"70, carente de toda potencialidad, ningún ser creado podría traer algo a la existencia, entendida en su sentido propio. Si carece de toda potencialidad es acto puro y, por lo tanto, carece de esencia en el sentido que hasta ahora se ha usado, es decir, como principio limitador del ser. Si se puede hablar de la esencia divina habría que decir que se trata de una "supraesencia"71, una 'esencia' que no limita el existir, sino que es su existir mismo. De este modo, desde el punto de vista de la línea entitativa se puede concluir lo siguiente:

\footnotetext{
69 Tomás de Aquino Suma Teológica, q. VII, a. 1.

7028 Para Finlayson, la esencia divina debe ser entendida como substantivo y verbo a la vez. C. Finlayson, Dios y la Filosofía 72.

71 C. Finlayson, Dios y la Filosofía 28.
} 
El sentido de la pregunta por los nombres de Dios en Pseudo Dionisio Areopagita y... 447

"Quad nos, aparece la existencia como el primer dato del ser, que nos
es revelado en nuestro proceso cognoscitivo. Conocemos los seres en
acto, existentes. Esta existencia es el acto del ser y ella en sí misma
no es sino actualidad. La conclusión, con respecto a Dios, ya desde
luego supuesta su existencia como Acto Puro, es evidente: la existen-
cia es el acto constitutivo formal de la Divinidad desde este punto de
vista entitativo. Y es también el primer aspecto metafísico de Dios
que se nos presenta"72.

No obstante, a este respecto Finlayson aclara que esta formalidad, si bien tiene un fundamento objetivo en la realidad divina, dice relación a nuestra inteligencia humana, con lo cual limita el sentido que hasta ahora parecía llevar la investigación. "Nuestro conocimiento de Dios es por vía de negación y analogía, de atribución y de trascendencia"73, de modo que los nombres divinos dicen más lo que Dios no es, que lo que Dios es. De allí que no sea difícil errar en ello, al confundir las diversas perspectivas posibles para abordar el problema. Desde la perspectiva de la línea entitativa, Dios es el que es, "es su ser" "4 es decir, en Él ser y esencia se identifican. Ahora bien, qué sea lo que eso significa en Dios y para Dios, es algo inconmensurable, pero como en las creaturas la esencia no se identifica con la existencia, es posible vislumbrar, por vía negativa, este primer nombre de Dios. Dios es el que es, pero el mismo lenguaje esconde una trampa al separar sujeto y predicado, propiamente Dios es, simplemente es su ser, es "Soy" 75 . Solo en Dios se da esta iden$\operatorname{tidad}^{76}$, esto lo separa radical y absolutamente de todo aquello que no se identifica con su ser. Los demás atributos de Dios se fundan en esta simplicidad radical, por cuanto la identidad de ser y existencia significa ser absoluto, es decir, no hay en Él el más mínimo resabio de nada y, por lo tanto, todo lo que significa ser y perfección se dicen también absolutamente de Dios.

El segundo nombre de Dios, según Clarence Finlayson, desde la perspectiva de su naturaleza, es 'pensamiento subsistente'. Dios ha sido comprendido como el existir subsistente, su esencia se identifica con su

\footnotetext{
72 C. Finlayson, Dios y la Filosofía 38.

73 C. Finlayson, Dios y la Filosofía 39.

74 Tomás de Aquino, De potentia Dei, q. 5, a. 8 (Cuadernos de Anuarios Filosófico, Pamplona 2005).

75 "La esencia divina es sustantivo y es verbo". C. Finlayson, Dios y la Filosofía 97.

76 C. Finlayson, Dios y la Filosofía 46.
} 
existencia, pero el existir mismo carece de todo contenido, ya que este viene por vía de la esencia. De modo que entendido Dios como puro existir y afirmada su absoluta trascendencia respecto de toda creatura, si bien esto revela su carácter infinito, se muestra así meramente como el reverso de una pura nada, es decir, con esto se revela como el que es absolutamente, pero si solo fuera esto, quedaría clausurado sobre sí mismo. "La existencia de suyo no nos dice nada específico" 77 . Por eso ahora Finlayson aborda el problema desde la perspectiva de la naturaleza divina, la cual intenta dar con el nombre de Dios en razón de su acción u operación. Desde una perspectiva metafísica, la operación expresa la quididad de la naturaleza, y en aquella esta cumple la virtualidad y potencialidad que contiene; la forma es, pues, principio de las operaciones. Ciertamente la operación participa de la actualidad de la existencia de un ser, pero no se identifica con la existencia misma. Si bien la existencia actualiza a la esencia, esta permanece potencial respecto de su misma existencia; los seres naturales deben realizar operaciones para conservarse en la existencia. De modo que "todo está predeterminado a la acción, y esta a la consecución de un fin"78. A esto lo llama Finlayson "el aspecto dinámico del ser"79, basándose en el adagio escolástico: a toda forma sigue una inclinación.

"El objeto formal de la metafísica es el ser real que alcanza su último cumplimiento en la existencia, este ser tiene una profunda realidad tendencional, un ontológico aspecto inclinativo y dinámico, inherente a su íntima esencialidad" 80 .

Por lo tanto, para conocer la naturaleza de las cosas es necesario recurrir a su actividad ${ }^{81}$, por cuanto la operación revela la quididad del ser. La esencia es principio limitativo de la existencia, de modo que todo ser natural es, pero no es absolutamente, de allí que para conservarse en la existencia no le baste ser, sino que tiene que realizar operaciones ordenadas precisamente a su conservación. La forma de esa actividad, que no tiene su principio inmediato en la existencia sino en la esencia, pone de manifiesto la naturaleza de ese ser. Como se puede ver, el punto de partida metafísico para desentrañar la naturaleza de la divinidad, parte

\footnotetext{
C. Finlayson, Dios y la Filosofía 83.

C. Finlayson, Dios y la Filosofía 74.

C. Finlayson, Dios y la Filosofía 75.

80 C. Finlayson, Dios y la Filosofía 75.

81 C. Finlayson, Dios y la Filosofía 77.
} 
El sentido de la pregunta por los nombres de Dios en Pseudo Dionisio Areopagita y... 449

por establecer la índole de las operaciones en las creaturas, como parece que no podría ser de otro modo, dada la naturaleza humana. Pero, esto mismo pone de manifiesto la radical insuficiencia del análisis, del que solo por analogía y muy imperfectamente puede hablarse de 'operación' en la naturaleza de Dios, no obstante, se debe tener presente que se trata de establecer tal naturaleza quoad nos.

"Si consideramos a Dios en cuanto naturaleza, o sea, en cuanto esencia como principio de operaciones o actividad, quoad nos, sostengo con Juan de Santo Tomás que no es la existencia la que se nos ofrece como principio de operaciones, como el primer atributo fundamental, sino la Intelección Actualísima"

Las condiciones que debe cumplir este nombre de Dios son las mismas que debía cumplir el primero: Debe ser propiedad exclusiva, de Dios, debe ser el principio de donde se deducen los demás atributos y distinto de ellos, debe ser principio de distinción entre Dios y las creaturas $^{83}$. El punto de partida de la demostración es la perfecta inmaterialidad y espiritualidad del Acto Puro. Es claro que, si bien en las creaturas al ser sigue una inclinación, aplicado esto a Dios, 'inclinación' solo indicaría" actividad inmanente y trascendentemente inmóvil" ${ }^{4}$, de modo que la actividad que procede de la forma divina no puede ser distinta de la Divinidad misma. Dios es puramente espiritual, ahora bien, en la creatura se dan dos formalidades espirituales que se distinguen realmente: inteligencia y voluntad ${ }^{85}$. En Dios, por el contrario, no puede darse esta distinción al modo de facultades diversas, sino que tanto su entender como su querer son actos inmanentes a su ser. Para Finlayson hay una precedencia real de la inteligencia respecto de la voluntad, que no explica, pero que puede presumirse que tiene un origen aristotélico. Para Aristóteles, el entendimiento es la facultad de los fines; solo una vez que se conoce el fin, interviene la voluntad ${ }^{86}$.

En Dios no existe potencialidad, de modo que la intelección divina no puede admitir separación entre sujeto y objeto, el acto por el que Dios se conoce a sí mismo es el mismo acto por el cual existe, de modo

\footnotetext{
82 C. Finlayson, Dios y la Filosofía 79.

83 C. Finlayson, Dios y la Filosofía 79.

84 C. Finlayson, Dios y la Filosofía 81.

85 C. Finlayson, Dios y la Filosofía 82.

86 Aristóteles, Ética a Nicómaco, VI, 2, 1139 a 25 (Centro de Estudios Constitucionales, Madrid 1994).
} 
que el ser inteligencia subsistente solo puede corresponder a Dios. Si el puro existir pone en evidencia lo que podríamos llamar la autoposición de Dios, el entendimiento subsistente revela la autoposesión de Dios, de tal modo que en Dios autoposición y autoposesión se identifican. De allí que la operación divina no puede considerarse un 'accidente ${ }^{87}$, ya que la operación divina no brota de un principio diverso de la operación misma, y no se distingue como acto de una potencia, porque en Dios no hay potencialidad ninguna. La distinción entre esencia y operación es solo producto de la limitación del entendimiento humano. Ciertamente entendimiento, intelección y esencia son los mismo en Dios; estrictamente hablando "la intelección actualísima no es una operación, sino la misma naturaleza" ${ }^{88}$. Pero, analógicamente, si la intelección actual es el principio de la actividad divina, entonces necesariamente de ella se siguen los demás atributos. Ahora bien, la intelección infinita piensa todo el universo, de modo que "las cosas son lo que son porque Dios las piensa" ${ }^{99}$, lo cual marca una diferencia radical y absoluta entre Dios y las creaturas. Así pues, "este intelligere es el existir divino en una perfecta y condensada identidad, íntima por ser totalmente transobjetiva e inmaterial" $"$.

El tercer nombre de Dios, según Finlayson, es Amor. Ahora bien, se debe tener presente que se trata, como él mismo señala, de un estudio metafísico, de modo que su punto de partida es el ente en cuanto tal. A este respecto, según Finlayson, la estructura de acto y potencia que se conforma a la de esencia y existencia, en los entes, pone de manifiesto que todo lo que es, es, desde sí mismo, dinámico, contiene en su raíz ontológica una tendencia, una inclinación ${ }^{91}$. El existir no es solamente un acto que instala a la cosa en la existencia, sino que en la íntima estructura del ente, la existencia se revela como un 'tensor', lo que, en otro lugar explica diciendo que "la esencia se mueve y opera para responder a la mayor actualidad o 'densidad ontológica' de la existencia”92. Así, como

\footnotetext{
87 C. Finlayson, Dios y la Filosofía 89.

88 C. Finlayson, Dios y la Filosofía 95 nota.

89 C. Finlayson, Dios y la Filosofía 91.

90 C. Finlayson, Dios y la Filosofía 98.

91 C. Finlayson, Dios y la Filosofía 99.

92 C. Finlayson, "Fundamentación de una metafísica dinámica", en Hombre, Mundo y Dios. Visión cristiana de la existencia (Cosmos, Bogotá 1953) 195-259.
} 
El sentido de la pregunta por los nombres de Dios en Pseudo Dionisio Areopagita y... $\mid 451$

se señalaba más arriba, a toda forma sigue una inclinación ${ }^{93}$, esa inclinación es hacia la completud existencial del propio ser, y por ello ser y bien se identifican, porque el ser tiene razón de fin. "Bonum est causa amoris propira, ut proprium ejus objectum " ${ }^{\text {' }}$. El bien existe, pues, en todos los seres y ellos tienden a la bondad, pero además, todos los seres se correlacionan entre sí en el intento del fin y, de este modo, en algún sentido todo ser es bueno para otro, y tal es el fundamento del orden cósmico ${ }^{95}$.

En los seres racionales, la base de su relación con el bien radica en el conocimiento, no obstante la raíz de este dinamismo hacia el bien es la existencia misma. El amor es la forma más perfecta de apetito por el bien que es ser en plenitud y, como pertenece al ámbito de la existencia, expresa una cierta superabundancia del ente ${ }^{96}$. Todo ser tiende a una conjunción de actividad y esencia, a unificar la acción con el ser íntimo propio, pero además tiende, a partir de esta unidad, a sobrepasarse a sí mismo. Y esta superabundancia se caracteriza en primer lugar por una radical tendencia a la comunicabilidad de sí. "El ente tiende y lucha por comunicar o expresar el íntimo secreto de su ser, el incomunicable e inefable misterio de su estructura" ${ }^{\prime 7}$. De allí que sea en el amor donde se revela, en los seres espirituales, la condición de persona. Efectivamente, el amor es esencialmente una forma de intimidad tal que, aunque no se supera el límite de la alteridad, sí cada uno se trasciende a sí mismo. Y, en segundo lugar, por ser el amor tendencia a una plenitud existencial, se caracteriza por una tendencia, no menos radical, a realizar el amor en una obra. El amor se revela como una tendencia ${ }^{98}$, de allí que el amor sea el acto por el que una esencia se prolonga y perfecciona realizando sus posibilidades 99 . El amor es existencial y concreto, se dirige a un objetofin que opera como tensor existencial y pone de manifiesto el dinamismo entitativo que caracteriza a todo ser. "El amor es la expresión más alta del dinamismo de la esencia, término final de la actividad, manifestación última de la riqueza y autonomía del ente" ${ }^{100}$. En este sentido el

\footnotetext{
93 J. De Santo Tomás, Cursus Philosophicus, t. III (Marietti, Turin 1930) 523.

94 Tomás de Aquino Suma Teológica, S. Th., I-II, q. 27, a. 1; q. 29 a. 1.

95 C. Finlayson, Dios y la Filosofía 111.

96 C. Finlayson, Dios y la Filosofía 107.

97 C. Finlayson, Dios y la Filosofía 113.

98 C. Finlayson, Dios y la Filosofía 117.

99 C. Finlayson, Dios y la Filosofía 119.

100 C. Finlayson, Dios y la Filosofía 148.
} 
amor concierta en esa superabundancia a la existencia y al pensamiento, por cuanto el amor realiza lo que conoce.

Así, pues, la argumentación de Finlayson sigue el siguiente derrotero: el amor nace de una esencial y radical necesidad de sobrepaso. El amor expresa la acción como la intensificación del ser, en su anhelo de sobrepaso. Un ser, cuya esencia es puro amor, es por naturaleza infinito. "Un Puro Amor será su propio ser íntimo, sobrepasado en sí mismo, ya que el mismo sobrepaso existencial no admite límite"101. En Dios, su Amor es expresión de una superabundancia infinita y, por ello, pone de manifiesto su Gloria, también infinita. Este nombre, según Finlayson, es "el más sublime y el más escondidamente secreto"102, y la más alta verdad que podemos alcanzar, de allí que el camino del amor sea el que sigue la vía mística. Para Finlayson es imposible concebir la existencia de una inteligencia pura sin estar acompañada de una voluntad o capacidad de $\operatorname{amar}^{103}$. Asimismo, el existir revela al ser 'hacia afuera', lo cual obliga a preguntar por el 'para qué' de esa revelación, es decir, por su 'intencionalidad trascendental': "al revelar al ser esencial revela la gloria y el amor de la Divinidad"104. De modo que en el amor se encuentran, desde la perspectiva de la creatura, los dos primeros nombres de Dios: existencia y entendimiento subsistentes:

"Consideramos al plano del Amor Divino como una super-existencia, implicada en la existencia divina, que expresa, del modo más íntimo y secreto, la actividad contemplativa del Espíritu, del Ser Supremo. Así el Amor de Dios nos aparece como una Pura Contemplación Existencial"105.

En el amor se conjugan, pues, existencia y entendimiento, tanto en las creaturas como, analógicamente, en la divinidad. El punto de partida no es meramente soy, o pienso, sino una voluntad previa que conduce el pensar hacia el ser, y es en esa voluntad donde se encuentran. Pero no se trata de una voluntad de dominio, o de apropiación, sino de una voluntad que busca una plenitud en el orden del ser; el amor consiste esencialmente en esa búsqueda, y tal ansia de ser pleno es connatural a todo

\footnotetext{
101 C. Finlayson, Dios y la Filosofía 150.

102 C. Finlayson, Dios y la Filosofía 154.

103 C. Finlayson, Dios y la Filosofía 18.

104 C. Finlayson, Dios y la Filosofía 28.

105 C. Finlayson, Dios y la Filosofía 37.
} 
ser creado. En Dios, esa plenitud "expresa la Gloria del Ser Infinito"106, como una generosidad y superabundancia de sí, de tal modo que "el fin de la creación es manifestar la gloria del Espíritu"107.

\section{CONCLUSIÓN}

Ciertamente para un tomismo riguroso, Finlayson ha ido más allá de los límites y capacidades del ser humano, al pretender establecer la esencia divina a partir de la sola razón. Si bien cada vez que da con uno de los nombres de Dios que expresarían su esencia, advierte que es solo por vía analógica y trascendentalmente que este se le puede aplicar a la divinidad, no obstante, afirma que no se trata de meros atributos ${ }^{108}$, sino nombres que expresan la esencia divina y cuyos atributos se definen a partir de estas determinaciones esenciales. En realidad, la investigación sobre los nombres de Dios que realiza Tomás de Aquino se inscribe más bien en la tradición de Pseudo-Dionisio, por cuanto se trata de establecer los atributos de Dios que se alcanzan por la doble vía catafática y apofática, ya que según sus propias palabras se nombra a Dios "por vía de eminencia y eliminación"109. Y, si bien los "nombres positivos expresan la substancia divina"110, estos los alcanzamos a partir de las creaturas, de modo que no lo pueden representar como una cosa del mismo género o especie, sino como el Principio supremo, de allí que todos los atributos, igualmente, que indican positivamente perfecciones, expresan la esencia divina ${ }^{111}$. Solo el nombre 'El que es' tiene el privilegio de ser el que es más principalmente propio de $\operatorname{Dios}^{112}$. Con lo cual se puede ver lo arriesgado del propósito de Finlayson, al intentar establecer la esencia metafísica de Dios y deducir de esta los atributos, como tales.

\footnotetext{
106 C. Finlayson, Dios y la Filosofía 159.

107 C. Finlayson, Dios y la Filosofía 134.

108 C. Finlayson, Dios y la Filosofía 48.

109 Tomás de Aquino Suma Teológica, q. 13, a. 1.

110 Tomás de Aquino Suma Teológica, q. 13, a. 2

111 Tomás de Aquino Suma Teológica, q. 13, a. 2.

112 Tomás de Aquino Suma Teológica, q. 13, a. 11.
} 
Por otra parte, se debe tener presente a este respecto la clásica distinción ${ }^{113}$ de San Agustín de Hipona entre "fides qua creditur" y "fides quae creditur" ${ }^{\prime 14}$ :

"Decimos con plena verdad que la fe impresa en los corazones de los creyentes, que estos mismos creen, proviene de una determinada doctrina; pero una cosa es lo que se cree [quae creduntur], y otra la fe por la cual se cree [qua creduntur]. Lo que se cree son verdades con existencia en el pasado, en la actualidad o en el futuro; la fe radica en el alma del creyente y es solo visible al que la posee; porque, si bien existe en otros, ya no es la misma, sino otra muy semejante" 115 .

Fides qua creditur es la dimensión subjetiva de la fe, fides quae creditur, es la dimensión objetiva, el contenido de esa fe; estrictamente no se distinguen entre sí como dos actos diversos, no hay fe sin contenido, ni tampoco un contenido abstracto sin el acto de asentimiento como tal. De modo que estos no significan dos elementos propiamente diversos, sino que uno se alimenta del otro, lo que se cree insta al acto de asentimiento, y el acto de asentimiento es una autodisposición respecto de lo que se cree.

Así, pues, la fuente originaria del propósito, aparentemente desproporcionado, de dar con los nombres divinos, debe ser indagado en la naturaleza misma de la fe. A la fe no le es suficiente la existencia de Dios, requiere, como decíamos, un contenido, un nombre, pero un nombre que sea efectivamente principio motivante de la fe, carácter este que no cumple, por sí sola, la mera existencia; 'hay Dios' ciertamente pone a la divinidad entre las cosas existentes, pero eso no significa de suyo que esté comprometido ni con el mundo ni con el ser humano en particular. Tampoco el entendimiento infinito y subsistente puede ser principio, por sí solo de la fe, por cuanto el acto de entender es de hecho inmanente al que entiende. Por ello, como sostiene Finlayson, el Amor es el supremo nombre de $\operatorname{Dios}^{116}$, porque solo en el amor Dios se hace presente a la fe como término real del ser humano en el orden existencial. También para Dionisio, y no es mera coincidencia, el encuentro de Dios con la creatura se realiza en el amor. Por ello, para Pseudo Dionisio, si

113 Guido Bausenhart, Einführung in die Theologie (Herder, Freiburg 2010) 306-307.

114 San Agustín, "Tratado de la Santísima Trinidad", XIII, 2, 5, en Obras de San Agustín, T. V. (BAC, Madrid, 1956) 706.

115 San Agustín, "Tratado de la Santísima Trinidad", XIII, 2, 5, en Obras de San Agustín, T. V. (BAC, Madrid, 1956) 706.

116 C. Finlayson, Dios y la Filosofía 159. 
El sentido de la pregunta por los nombres de Dios en Pseudo Dionisio Areopagita y... $\mid 455$

'Bien' es el nombre más propio de Dios, esto es así porque el bien es el principio teleológico del amor. En realidad, no se trata meramente de que Dios sea bueno, sino que es bueno para la creatura, es bueno para mí, y tal sería el mensaje de las Sagradas Escrituras, particularmente del Nuevo Testamento. Pseudo Dionisio ha sido maestro de la mística precisamente porque esta se dirige a un Dios personal pleno de contenido y de sentido. De allí que el camino que recorre los nombres de Dios sea una vía existencial y no meramente especulativa.

El punto de partida para develar los nombres de Dios en Pseudo Dionisio es la Revelación, porque en ello se pone en evidencia el radical compromiso de Dios con la creatura, que tiene el carácter de un llamado amoroso, como que es el bien de esta, y, a la vez, tiene también el carácter de un camino por vía de la contemplación. En Finlayson, la aproximación metafísica a la pregunta que interroga por los nombres de Dios pretende dar cuenta de Dios en nosotros, en la medida que la razón hace presente una tendencia radical al desborde de sí. En ambos casos Dios manifiesta su carácter personal y fundamento de una existencia humana plena de sentido.

Así, pues, lo que en términos metafísicos se muestra, según Finlayson, como existencia subsistente, entendimiento subsistente y amor como gloria del Ser Infinito (del mismo modo como la meditación de los nombres de Dios de la Sagrada Escritura), si bien son el término de una exploración estrictamente metafísica, no obstante pueden ser también comprendidos como enraizados en una experiencia vital. Por eso los nombres que Finlayson propone, creo que deben ser comprendidos, desde esta perspectiva; y tales serían lo que me atrevería a llamar 'los tres momentos fundamentales de la fe': Dios me revela su existencia, me conoce, me ama, y en ese orden. Naturalmente esto significa llevar el "quoad nos" más allá del ámbito puramente especulativo. Pero Finlayson insiste en reiteradas oportunidades ${ }^{117}$ en el carácter existencial de la filosofía tomista, y esto en un doble sentido; por una parte metafísico, es decir, el núcleo metafísico de la filosofía tomista es el ser como acto; pero, por otra parte, esta filosofía también da cuenta, según Finlayson, de la existencia humana tendida radicalmente hacia su plenitud. De este modo no creo que entender la esencia de Dios en primera persona (existe, me conoce, me ama) signifique en absoluto traicionar su pensamiento. 'Dios me revela su existencia' pone a la propia vida frente a sus limitaciones y precariedad, cual es el punto de partida

117 C. Finlayson, Dios y la Filosofía 7, 32, 118, 144, 205. 
de la fe. 'Dios me conoce' revela el carácter espiritual ${ }^{118}$ de Dios, lo cual significa que no está clausurado sobre sí, sino abierto al universo entero ${ }^{119}$. Dios me ama, constituye un llamado, una vocación de absoluto y es, de este modo, el punto culminante de la fe, ya que pone de manifiesto el carácter personal de la divinidad ${ }^{120}$. Finlayson, citando a Unamuno dice "gracias al amor sentimos todo lo de carne que tiene el espíritu"121. El amor de Dios pone al ser humano individual ${ }^{122}$ frente a sí mismo de tal manera que entonces no solo percibe su propio valor, sino que sobre todo invita a la realización plena de ese valor. Finlayson afirma, con un tono más propio de Pseudo Dionisio, que "el doble proceso de ascensión de las creaturas hacia Dios y del descenso de su Bondad a ellas funda la armonía dinámica del universo que cubre y enlaza a su vez a todos los seres suspendidos en su principio"123.

De este modo, estos tres momentos fundamentales de la fe, "Dios me revela su existencia, me conoce y me ama", correlatos vivenciales de la esencia divina tal como Finlayson la expone, dejan en evidencia que el contenido subjetivo de la fe, aquello por lo que creemos, y aquello que creemos constituyen en el creyente un acto indisoluble. El camino metafísico de Finlayson no culmina en absoluto en el "motor inmóvil", sino en un Dios personal que es, a la vez, principio motivante y contenido de la fe.

Se puede afirmar que hay una cercanía fundamental entre Finlayson y Pseudo Dionisio, pese a la aparente intensión puramente especulativa del escrito del primero, y a la intención apologética del segundo, lo cual se puede ver, también, en los permanentes giros poéticos y existenciales a los que recurre Finlayson cuando el lenguaje, como él mismo señala, resulta insuficiente ${ }^{124}$. Pero, sobre todo, Pseudo Dionisio y Clarence Finlayson intentan dar con el contenido de la fe, de modo que esta sea un 'tensor', en palabras de Finlayson, que revele al ser humano su identidad al mismo tiempo que su vocación.

118 C. Finlayson, Dios y la Filosofía 80.

119 C. Finlayson, Dios y la Filosofía 111.

120 C. Finlayson, Dios y la Filosofía 116.

121 C. Finlayson, Dios y la Filosofía 110.

122 C. Finlayson, Dios y la Filosofía 121.

123 C. Finlayson, Dios y la Filosofía 113.

124 C. Finlayson, Dios y la Filosofía 72. 